\title{
General Procedure for Formulation of Multiaxial Fatigue Failure Criteria in Frequency Domain
}

\author{
Adam Niesłony ${ }^{1,1}$, Michał Böhm ${ }^{1}$, Robert Owsiński ${ }^{1}$ \\ ${ }^{1}$ Opole University of Technology, Faculty of Mechanical Engineering, Department of Mechanics and \\ Machine Design, Opole, Poland
}

\begin{abstract}
Fatigue life assessment under multiaxial loading conditions needs the use of multiaxial fatigue failure criteria. There are many formulations of such criteria which are usually divided in the groups of critical plane criteria and invariants based criteria. Other classifications refer to the parameter that determines fatigue, and these are stress, strain and energy criteria. Recently, the development of computational techniques for fatigue analysis in the frequency domain have been noticed. Analyzing the literature, it can be noted that these criteria are mainly created by the new formulation of them in the new domain. There is therefore a need to develop a general approach to the problem of formulating criteria in the frequency domain on the basis of existing proposals for the time domain. The paper presents the manner in which the values appearing in the criteria in the frequency domain can be determined. In particular, the process of formulating criteria of multiaxial random fatigue, using the critical plane approach proposed by Macha was presented. During the formation the linearity of such criteria was successfully used. Situations that indicate the correctness of transformations are also presented.
\end{abstract}

\section{Introduction}

The idea of multiaxial fatigue failure criteria use is over one hundred years old. Nevertheless it is still an issue due to the fact that still new criteria are developed. Mean stress, phase shift between shear and axial stress etc. are undergoing many modifications in these criteria. Many scientists over the years have formed different ways to take into account the multiaxial stresses. A big part of those methods is defined with the use of the critical plane approach. In this approach we are searching for the most damaged plane with the use of parameters defined along directions in the Cartesian coordinate system inside of our object. A large literature review in the terms of critical plane criteria can be found in the paper by Karolczuk and Macha [10] or Carpinteri et al. [6]. The objective of this work is to present the procedures behind the creation of multiaxial fatigue failure criteria in frequency domain with the use of criteria defined in the time domain. The frequency domain calculations still have many drawbacks in comparison with time domain calculations, but the indisputable fact is that in some cases we are possessing only the frequency definition of loading (PSD of loading) and that the frequency calculations are faster [1]. The recent development in computer science and hardware accelerated calculations has allowed us to analyze large objects with an ultra-high amount of computation power. Nevertheless time

${ }^{1}$ Corresponding author: a.nieslony@po.opole.pl 
domain calculations are still a lot slower in comparison to frequency, even with the mentioned high-end equipment. The basic scientific justification for the presentation of this methodology lies in the fact, that many time domain multiaxial criteria can be easily adapted to the frequency domain. The interesting part of this paper shows the relation between constants and variables that can be defined in different ways but have the same interpretation in both time and frequency domain. The reader should pay attention to the criteria domain and its expression type (strain, stress) and the comments regarding the possible application in frequency domain. The conclusion and discussion part of the paper shows the example of a simple criteria transformed into frequency domain and the dangers of the use of such criteria in comparison to time domain.

In the spectral analysis our aim is to describe the power distribution of a signal. In the case of fatigue the signal can be the stress or strain history registered during fatigue tests [17]. This is usually obtained with the use of the power spectral density (PSD) functions $G(f)$. In case of a random stress vector in the form

$$
\sigma(t)=\left[\begin{array}{llllll}
\sigma_{x x} & \sigma_{y y} & \sigma_{z z} & \tau_{x y} & \tau_{y z} & \tau_{z x}
\end{array}\right]
$$

the matrix of PSD functions is presented as following [13]:

$$
\mathbf{G}_{\sigma}(f)=\left[\begin{array}{ccc}
G_{11}(f) & \cdots & G_{16}(f) \\
\vdots & \ddots & \vdots \\
G_{61}(f) & \cdots & G_{66}(f)
\end{array}\right],
$$

where each $G_{i j}(f)$ is the one sided power spectral density function for $i, j=1 \ldots 6$ of the component of stress determined for frequencies $f \geq 0$. For a multiaxial strain, we can directly use the matrix of PSD functions of strain $\mathbf{G}_{\varepsilon}(f)$ obtained in accordance with the previous method of notation using strain vector as following:

$$
\varepsilon(t)=\left[\begin{array}{llllll}
\varepsilon_{x x} & \varepsilon_{y y} & \varepsilon_{z z} & \varepsilon_{x y} & \varepsilon_{y z} & \varepsilon_{z x}
\end{array}\right] .
$$

\section{Selected time domain multiaxial fatigue failure criteria}

\subsection{Strain criteria}

Many strain criteria have been formed over the years, but none has been defined for both domains beside the criteria by Macha [12]. The criteria defines the position of the critical plane in which the damage gives the highest results. For example the criterion of maximum shear strains in the fracture plane takes the form

$$
\varepsilon_{e q}(t)=\frac{1}{1+v}\left[\begin{array}{l}
\left(l_{1}^{2}-l_{3}^{2}\right) \varepsilon_{x x}(t)+\left(m_{1}^{2}-m_{3}^{2}\right) \varepsilon_{y y}(t)+\left(n_{1}^{2}-n_{3}^{2}\right) \varepsilon_{z z}(t)+2\left(l_{1} m_{1}-l_{3} m_{3}\right) \varepsilon_{x y}(t)+ \\
+2\left(m_{1} n_{1}-m_{3} n_{3}\right) \varepsilon_{y z}(t)+2\left(l_{1} n_{1}-l_{3} n_{3}\right) \varepsilon_{z x}(t)
\end{array}\right]
$$

where:

$\varepsilon_{\mathrm{xx}}(t), \varepsilon_{\mathrm{yy}}(t), \varepsilon_{\mathrm{zz}}(t), \varepsilon_{\mathrm{xy}}(t), \varepsilon_{\mathrm{yz}}(t), \varepsilon_{\mathrm{zx}}(t)$ - components of the strain tensor, see vector (3), $m_{k}$, $n_{k}$, $l_{k}$ for $k=1,2,3$ - direction cosines of averaged directions of principal strains, $v$ poissons ratio. 


\subsection{Stress criteria}

As we analyze the literature we can clearly see that a huge amount of criteria has been formed with the use of stress description. This paragraph focus is set on the presentation of these criteria that can be also described in the frequency domain. The first model that is being described and is also the legacy of professor Macha's [11] work, started with his habilitation thesis where he defined the criteria for multiaxial fatigue within the stress framework with the use of the critical plane

$$
\sigma_{\eta}(t)=l_{1}^{2} \sigma_{x x}(t)+m_{1}^{2} \sigma_{y y}(t)+n_{1}^{2} \sigma_{z z}(t)+2 l_{1} m_{1} \sigma_{x y}(t)+2 m_{1} n_{1} \sigma_{y z}(t)+2 l_{1} n_{1} \sigma_{z x}(t) .
$$

Matake [14] has formed a shear stress criteria that coincides with Gough empirical formula for brittle materials under combined stress

$$
\frac{\tau_{n a}+\alpha\left(\sigma_{n m}+\sigma_{n a}\right)}{\beta} \leq 1,
$$

where: $\tau_{n a}$ - shear stress, $\sigma_{n a}$ - normal stress, $\sigma_{n m}$ - mean normal stress, $\alpha$ and $\beta$ are material properties given by:

$$
\left\{\begin{array}{l}
\alpha=\left(2 t_{-1} / f_{-1}\right)-1 \\
\beta=t_{-1}
\end{array},\right.
$$

where $f_{-1}$ and $t_{-1}$ are the endurance limits for a reversed tension stress and a reversed torsion stress.

Crossland [7] formulated a stress criteria that used the amplitude of the stress deviator with the assumption of zero mean stress

$$
\frac{\sqrt{J_{2, a}}+\alpha \max [p(t)]}{\beta} \leq 1,
$$

where $J_{2, \mathrm{a}}$ is the amplitude of the second invariant of the stress deviator which is given for zero-mean stresses.

Carpinteri et al [5] have formed a stress criteria with the use of descriptors depending on the fatigue limits for normal and shear stress

$$
\sigma_{e q}=\sqrt{\sigma_{\max }^{2}+\left(\frac{\sigma_{a f,-1}}{\tau_{a f,-1}}\right)^{2} \tau_{a}^{2}},
$$

where:

$\sigma_{\max }$ - maximum normal stress vector, $\tau_{a}$ - shear stress vector amplitude, $\sigma_{a f,-1}$ - fully reversed fatigue limit for normal stress for the ratio $R=-1, \tau_{a f,-1}$ - fully reversed fatigue limit for shear stress for the ratio $R=-1$.

Susmel's [20] approach to multiaxial fatigue criteria was set on the assumption of a relationship between the maximum stress and the shear stress amplitude. This factor has been used in the description of the reference shear stress with the use of the fatigue limit for normal and shear stress: 


$$
\begin{gathered}
\rho=\left(\frac{\sigma_{\max }}{\tau_{a}}\right), \\
\tau_{r e f}(\rho)=\left(\frac{\sigma_{a f=-1}}{2}-\tau_{a f=-1}\right) \rho+\tau_{a f=-1} .
\end{gathered}
$$

\section{Frequency domain multiaxial fatigue criteria}

The frequency domain fatigue criteria set for the multiaxial case have a short history. The most famous one has been presented by Pitoiset et al. [19] and is using the modification of the Huber-Mises criteria defined for the static case. The creators of this formula noticed, that some of the basic constants and variables used in the time domain have their representation in the frequency domain. Nevertheless the intention of authors of this paper is to show the criteria that have been developed from time to frequency domain. The criteria have been divided into two groups, which deal with strain and stress criteria.

\subsection{Strain criteria}

In his book Macha [12] has proposed the extension of his criteria to frequency domain with a simple transformation for linear systems, known from the spectral analysis of stochastic processes. Such transformation is eligible because the form of multiaxial fatigue failure criteria proposed by Macha are linear. The criterion Eq. (4), presented as an example in this paper, can be rewritten as

$$
\varepsilon_{e q}(t)=\sum_{k=1}^{6} a_{k} \varepsilon_{k}(t)
$$

with the definition of coefficients $a_{k}$ presented in Table 1 and the components of the strain vector Eq. (3). According to the mentioned transformation of linear system PSD of equivalent strain can be obtained from the components of $\mathbf{G}_{\varepsilon}(f)$

$$
G_{e q}(f)=\sum_{i=1}^{6} \sum_{j=1}^{6} a_{i} a_{j} G_{i j}(f)=\sum_{i=1}^{6}\left[a_{i}^{2} G_{i i}(f)+2 \sum_{j=i+1}^{6} a_{i} a_{j} G_{i j}(f)\right] .
$$

Some of the extensions of the strain criteria has been shown by Niesłony and Böhm in connection with fatigue failure analysis supported with finite element method [3,14].

\subsection{Stress criteria}

The same rule as in the case of the strain criteria by Macha [13] has been applied for his approach for stress criteria

$$
\sigma_{e q}(t)=\sum_{k=1}^{6} a_{k} \sigma_{k}(t)
$$

with the definition of coefficients $a_{k}$ presented in Table 1 and the components of the stress vector Eq. (1). PSD of equivalent stress is computed using $\mathbf{G}_{\sigma}(f)$ according to Eq. (13). 
Table 1. Coefficients $a_{k}$ in $\varepsilon_{e q}(t)$ and $\sigma_{e q}(t)$ expressions

\begin{tabular}{|l|c|c|}
\hline $\mathbf{a}$ & Eq. (4) & Eq. (5) \\
\hline$a_{1}$ & $\frac{l_{1}^{2}-l_{3}^{2}}{1+v}$ & $l_{1}^{2}$ \\
\hline$a_{2}$ & $\frac{m_{1}^{2}-m_{3}^{2}}{1+v}$ & $m_{1}^{2}$ \\
\hline$a_{3}$ & $\frac{n_{1}^{2}-n_{3}^{2}}{1+v}$ & $n_{1}^{2}$ \\
\hline$a_{4}$ & $\frac{2\left(l_{1} m_{1}-l_{3} m_{3}\right)}{1+v}$ & $2 l_{1} m_{1}$ \\
\hline$a_{5}$ & $\frac{2\left(m_{1} n_{1}-m_{3} n_{3}\right)}{1+v}$ & $2 m_{1} n_{1}$ \\
\hline$a_{6}$ & $\frac{2\left(l_{1} n_{1}-l_{3} n_{3}\right)}{1+v}$ & $2 l_{1} n_{1}$ \\
\hline
\end{tabular}

Pitoiset et al. [19] have redefined the criterion proposed by Crossland

$$
\frac{\sigma_{1} F\left(2 N_{1}\right) / \sqrt{3}+\alpha \sigma_{p} F\left(N_{2}\right)}{\beta} \leq 1,
$$

Pitoiset et al. [19] have also redefined the criterion proposed by Matake:

$$
\frac{\sigma_{1}^{*} F\left(N_{1}^{*}\right)+\alpha \sigma_{n}^{*} F\left(N_{2}^{*}\right)}{\beta} \leq 1,
$$

where $\sigma_{n}=\left[\sqrt{\xi_{0}}\right], \xi_{0}$ is the zero spectral moment.

Carpinteri [4] has proposed a modification of his stress criteria in terms of use in the frequency domain

$$
G_{e q}=G_{w, w}+\left(\frac{\sigma_{a f,-1}}{\tau_{a f,-1}}\right) G_{v w, v w}
$$

where: $G_{w, w}$ - PSD function of normal stress $\sigma_{\mathrm{w}}, G_{v \mathrm{w}, v \mathrm{w}}$-PSD function of shear stress $\tau_{v w}$.

Susmel et al. [21] have taken a similar approach by taking the $\rho$ factor into consideration and based their reference criteria on the basis of fatigue limits expressed in the form of square roots of fatigue limits of invariants:

$$
\sqrt{J_{2 A \rho_{\text {ref }}}}=\left(\sqrt{J_{2 A \rho=-1}}-\sqrt{J_{2 A \rho=0}}\right) \rho+\sqrt{J_{2 A \rho=0}},
$$


where: $\sqrt{J_{2 A \rho=-1}}, \sqrt{J_{2 A \rho=0}}$ - fatigue limits expressed in terms of amplitude of the square root of the second invariant of the stress deviator.

\section{Switching from time to frequency domain}

As mentioned in the earlier paragraphs the goal of this paper is to show the procedures which are used to obtain parameters, constants and variables defined in the time domain that can be used indirectly or directly in the frequency domain. In order to explain the parameters used in the frequency domain that can be withdrawn from the time domain we need to understand the basic nomenclature, as well as laws and formulas used mainly in the stochastic science.

\subsection{Power spectral density}

Power spectral density of the signal describes the overall structure of a random process using the frequency spectral density of mean values of the physical signal in question. This value can be determined for the interval from $f$ to $f+\Delta f$ using a central-pass filter with a narrow band and averaging the square on the output of the filter [2]:

$$
G_{x}(f)=\lim _{\Delta f \rightarrow 0} \frac{\Psi_{x}(f, \Delta f)}{\Delta f}=\lim _{\Delta f \rightarrow 0} \frac{1}{\Delta f}\left[\lim _{T \rightarrow \infty} \frac{1}{T} \int_{0}^{T} x^{2}(t, f, \Delta f) d t\right] .
$$

where: $\Psi_{x}$ - the mean square value of the process $x(t), T$ - time of observation, $x(t, f, \Delta f)-$ component of $x(t)$ in the frequency range from $f$ to $f+\Delta f$. For small values of $\Delta f$ the formula (Eq.19) shows the one-sided PSD function. The power spectral density has no information about the phase of each frequency component, but in the case of multi-load, i.e. $\mathbf{G}_{\sigma}(f)$ and $\mathbf{G}_{\varepsilon}(f)$, the power spectral density matrix contains information on the mutual phase of the load components.

\subsection{Spectral moments}

The spectral moments that are usually used are up to the 5th one for calculations $\xi_{k}$ $(k=0, \ldots, 4)$ and can be defined on the basis of the PSD with the given formula [17]:

$$
\xi_{k}=\int_{0}^{\infty} G_{x}(f) f^{k} d f
$$

The zero (first) spectral moment is the variance

$$
\xi_{0}=\mu_{2} .
$$

Up to the fourth moment we can use variations of combinations of moments which can be used to describe certain parameters of the loading conditions:

- spectral bandwidth:

$$
S B=\sqrt{\frac{1-\xi_{2}^{2}}{\xi_{0} \xi_{4}}},
$$


- number of up-crossings of level zero:

$$
N_{0}=\sqrt{\frac{\xi_{2}}{\xi_{0}}},
$$

- expected number of peaks within a unit of time:

$$
M^{+}=\sqrt{\frac{\xi_{4}}{\xi_{2}}} .
$$

\subsection{Mean stress}

Mean stress has a huge impact on the fatigue life. Nevertheless there are no solutions to take it into account in the frequency domain for the multiaxial case. There are some solutions related to the uniaxial calculations proposed by Niesłony and Böhm [16]. They have proposed to use a transformation function in order to take the mean stress into account:

$$
G_{\sigma T}(f)=\left[K\left(\sigma_{m}, P\right)\right]^{2} G_{\sigma}(f)
$$

where: $G_{\sigma}(f)$ is the power spectral density of a centered stress course $\left[\sigma(t)-\sigma_{m}\right], K$ - is the coefficient defined with the use of an appropriate mean stress compensation model:

$$
K_{N B}=1+\sigma_{m}\left(\frac{1-R}{R+1}\right) \frac{\sigma_{a N, R=-1}-\sigma_{a N, R}}{\left(\sigma_{a N, R}\right)^{2}},
$$

where: $\sigma_{m}$ - mean stress, $\sigma_{a N, R=-1}$ and $\sigma_{a N, R}-$ stress amplitudes read off from S-N curves for alternating and for a chosen stress ratio $R$.

\subsection{Max values from PSD}

In some criteria there are maximum values from the analyzed course. Maximum values can also be evaluated in a statistical manner, as presented by Davenport [8, 9, 19]:

$$
E\left[\max _{0 \leq t \leq T}[x(t)]\right]=\sigma_{1}\left[\sqrt{2 \ln \left(N_{0} T\right)}+0.5772 / \sqrt{2 \ln \left(N_{0} T\right)}\right]
$$

Please note that the maximum value depends on time, which forces the optimization algorithm to be used during fatigue calculations.

\subsection{Stress state invariants and von Mises stress}

Pitoiset and Pneumont [18] described the equivalent von Mises stress method, where the PSD of equivalent stress is defined by:

$$
G_{e q M}(f)=\operatorname{tr}\left\{Q_{M} \mathbf{G}_{\sigma}(f)\right\}
$$

where for the spatial stress state 


$$
Q_{M}=\left(\begin{array}{cccccc}
1 & -1 / 2 & -1 / 2 & 0 & 0 & 0 \\
-1 / 2 & 1 & -1 / 2 & 0 & 0 & 0 \\
-1 / 2 & -1 / 2 & 1 & 0 & 0 & 0 \\
0 & 0 & 0 & 3 & 0 & 0 \\
0 & 0 & 0 & 0 & 3 & 0 \\
0 & 0 & 0 & 0 & 0 & 3
\end{array}\right)
$$

The PSD of stress deviator can be obtained from the PSD of von Mises stress:

$$
G_{s d}(f)=\frac{G_{e q M}(f)}{\sqrt{3}} .
$$

Hydrostatic pressure can be computed following the presented methodology, Eq. (28), and:

$$
Q_{h}=\frac{1}{3}\left(\begin{array}{cccccc}
1 & 1 & 1 & 0 & 0 & 0 \\
1 & 1 & 1 & 0 & 0 & 0 \\
1 & 1 & 1 & 0 & 0 & 0 \\
0 & 0 & 0 & 0 & 0 & 0 \\
0 & 0 & 0 & 0 & 0 & 0 \\
0 & 0 & 0 & 0 & 0 & 0
\end{array}\right) .
$$

\section{Observations and conclusions}

Many Scientists which were working on the creation process of multiaxial fatigue criteria have discovered the relation between the time and frequency domain between many constants and variables. Most of the relations still refer to the method of equivalent von Mises stress, which may be directly linked to the formulations of Power Spectral Density matrix. Another case is where we can directly link constants and variables obtained in the time domain to the use of spectral moments and the variance, which may be used in the process of fatigue life assessment. We can directly link the methods in the process of calculating:

- Equivalent stress amplitudes,

- Stress deviators,

- Variance,

- Expected extremes (maximum values over time).

The mentioned examples are by no means exhausting this topic, but serve as a platform for a broader discussion on the expansion of other popular criteria to frequency domain.

This work was supported by the National Science Centre, Poland, within the OPUS 12 grant, contract No. UMO-2016/23/B/ST8/03408.

\section{References}

1. A. Banvillet, T. Łagoda, E. Macha, A. Niesłony, T. Palin-Luc, J.-F. Vittori, Fatigue life under non-Gaussian random loading from various models, Int. J. Fatigue. 26: 349-363 (2004)

2. J.S. Bendat, Random data: analysis and measurement procedures, 4th ed, Wiley, Hoboken, N.J, (2010) 
3. M. Böhm, A. Nieslony, Strain-based multiaxial fatigue life evaluation using spectral method, in: J. Papuga, M. Ruzicka (Eds.), 3rd Int. Conf. Mater. Compon. Perform. Var. Amplitude Load. Val 2015, Procedia Engineering 101: 52-60 (2015)

4. A. Carpinteri, A. Spagnoli, C. Ronchei, D. Scorza, S. Vantadori, Critical Plane Criterion for Fatigue Life Calculation: Time and Frequency Domain Formulations, Procedia Eng. 101: 518-523 (2015)

5. A. Carpinteri, A. Spagnoli, S. Vantadori, A multiaxial fatigue criterion for random loading, Fatigue Fract. Eng. Mater. Struct. 26: 515-522 (2003)

6. A. Carpinteri, A. Spagnoli, S. Vantadori, A review of multiaxial fatigue criteria for random variable amplitude loads, Fatigue Fract. Eng. Mater. Struct. 40: 1007-1036 (2017)

7. B. Crossland, Effect of large hydrostatic pressures on the torsional fatigue strength of an alloy steel, Proc. Int. Conf. Fatigue Met 138-149 (1956)

8. A.G. Davenport, Note on the distribution of the largest value of a random function with application to gust loading., Proc. Inst. Civ. Eng. 28: 187-196 (1964)

9. A. Dua, M. Clobes, T. Höbbel, V. Matsagar, Dynamic Analysis of Overhead Transmission Lines under Turbulent Wind Loading, Open J. Civ. Eng. 05: 359-371 (2015)

10. A. Karolczuk, E. Macha, A Review of Critical Plane Orientations in Multiaxial Fatigue Failure Criteria of Metallic Materials, Int. J. Fract. 134: 267-304 (2005)

11. E. Macha, Mathematical models of the life to fracfture for materials subject to random complex stress systems, Wroclaw Tech. Univ. [in Polish], (1979)

12. E. Macha, ed., Generalization of Strain Criteria of Multiaxial Cyclic Fatigue to Random Loading, VDI Verlag, Düsseldorf, (1988)

13. E. Macha, Spectral method of fatigue life calculation under random multiaxial loading, Mater. Sci. 32: 339-349 (1996)

14. T. Matake, An Explanation on Fatigue Limit under Combined Stress, Bull. JSME. 20: 257-263 (1977)

15. A. Nieslony, M. Böhm, Application of Spectral Method in Fatigue Life Assessment Determination of Crack Initiation, J. Theor. Appl. Mech. 50: 819-829 (2012)

16. A. Niesłony, M. Böhm, Frequency-domain fatigue life estimation with mean stress correction, Int. J. Fatigue. 91: 373-381 (2016)

17. A. Nieslony, E. Macha, Spectral Method in Multiaxial Random Fatigue, Springer, (2007)

18. X. Pitoiset, A. Preumont, Spectral methods for multiaxial random fatigue analysis of metallic structures, Int. J. Fatigue. 22: 541-550. (2000)

19. X. Pitoiset, I. Rychlik, A. Preumont, Spectral methods to estimate local multiaxial fatigue failure for structures undergoing random vibrations, Fatigue Fract. Eng. Mater. Struct. 24: 715-727 (2001)

20. L. Susmel, Multiaxial fatigue limits and material sensitivity to non-zero mean stresses normal to the critical planes, Fatigue Fract. Eng. Mater. Struct. 31: 295-309 (2008)

21. L. Susmel, R. Tovo, Estimating fatigue damage under variable amplitude multiaxial fatigue loading, Fatigue Fract. Eng. Mater. Struct. 34: 1053-1077 (2011) 